\title{
Mechanisms for Flavin Mediated Oxidation: Hydride or Hydrogen-Atom Transfer?
}

\author{
Felipe Curtolo and Guilherme M. Arantes* \\ Department of Biochemistry, Instituto de Química, Universidade de São Paulo, Av. Prof. \\ Lineu Prestes 748, 05508-900, São Paulo, SP, Brazil \\ E-mail: garantes@iq.usp.br
}

\begin{abstract}
Flavins are versatile biological cofactors which catalyze proton-coupled electron transfers (PCET) with varying number and coupling of electrons. Flavin mediated oxidation of nicotinamide adenine dinucleotide $(\mathrm{NADH})$ and of succinate, initial redox reactions in cellular respiration, were examined here with multiconfigurational quantum chemical calculations and a simple analysis of the wave-function proposed to quantify electron transfer along the proton reaction coordinate. The mechanism of NADH oxidation is a prototypical hydride transfer, with two electrons moving concerted with the proton to the same acceptor group. However, succinate oxidation depends on the elimination step and can proceed through the transfer of hydride or hydrogen-atom, with proton and electrons moving to different groups in both cases. These results help to determine the mechanism of fundamental but still debated biochemical reactions, and illustrate a new diagnostic tool for electron transfer that can be useful to characterize a broad class of PCET processes.
\end{abstract}

\section{Introduction}

Enzymes equipped with flavin cofactors comprise the most abundant class of natural catalysts for combined proton and electron transfer. ${ }^{1,2}$ The redox center in all natural flavins is formed by the heteronuclear tricyclic isoallox- azine ring (Figure 1), primarily attached to the protein by noncovalent hydrogen bonds, stacking and cation- $\pi$ contacts. ${ }^{3-5}$ These interactions also modulate the flavin redox potential from -400 to $60 \mathrm{mV}$, allowing oxidation of a range of aliphatic and aromatic substrates. ${ }^{6-8}$

Flavin redox reactions are an example of proton-coupled electron transfers or PCET, a broad family of reactions and energy conversion processes in chemistry. ${ }^{9-11}$ PCET mechanisms are characterized by the number of electrons involved, such as in hydride $\left(2 \mathrm{e}^{-} / 1 \mathrm{H}^{+}\right)$ versus hydrogen-atom $\left(1 \mathrm{e}^{-} / 1 \mathrm{H}^{+}\right.$, or HAT $)$ transfer; ${ }^{12}$ the order of steps or their concurrency, such as electron transfer first, proton second (ETPT) or concerted proton-electron transfer (CPET); ${ }^{9,13}$ whether the transfers proceed from(to) the same or different chemical groups in the donor(acceptor), as in multiple-site PCET; ${ }^{10,14}$ the tunneling behavior and the adiabacity or participation of excited-states in the transfer processes. ${ }^{13,15}$

Thermochemical and redox potential measurements determined that flavins may undergo hydride and single-electron $\left(1 \mathrm{e}^{-}\right)$transfers, ${ }^{10,16}$ and molecular simulations explored their tunneling effects. ${ }^{17-20}$ But a description of possible HAT, particularly in enzymatic mechanisms, has received less attention. ${ }^{21} \mathrm{~A}$ remarkable example is the BLUF flavoprotein which has been shown by ultrafast spectroscopy and simulations to sustain HAT by light activation. ${ }^{22,23}$

Quantitative diagnostics of PCET mech- 

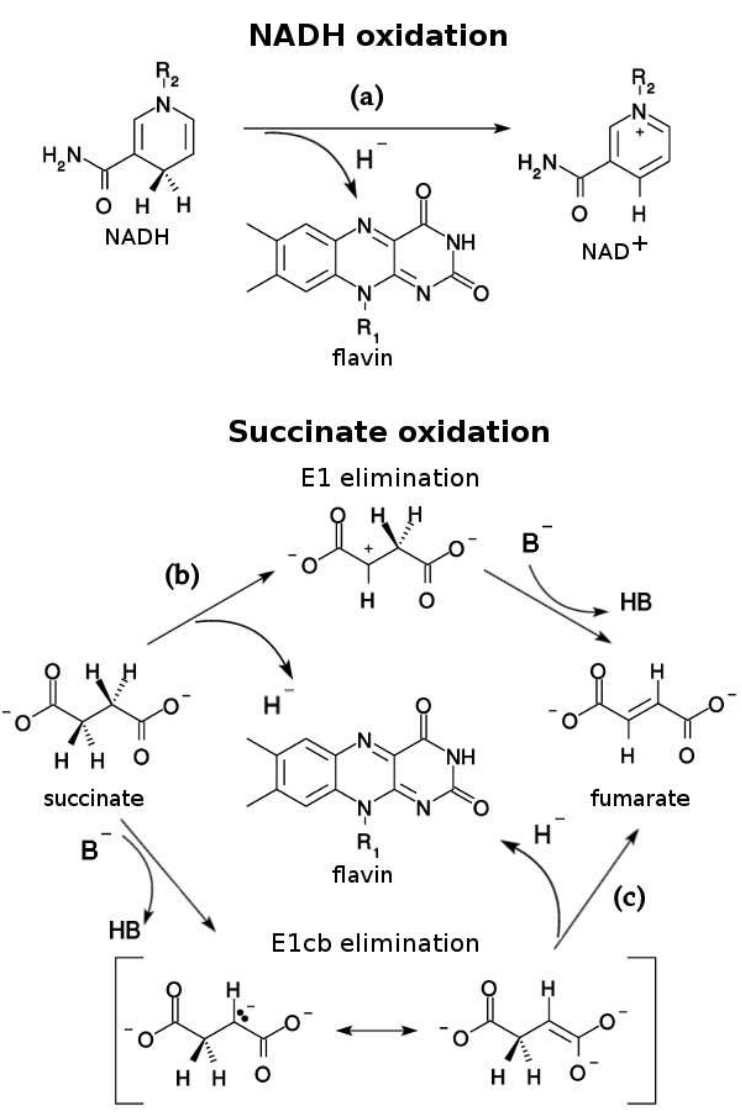

Figure 1: Flavins mediate oxidation of NADH and succinate. NADH reacts by elimination from the nicotinamide ring, step (a). Two reaction sequences are possible for succinate oxidation: unimolecular elimination (E1) is shown in step (b) and conjugate base elimination (E1cb) is in step (c). Biologically relevant flavins differ only in the $R_{1}$ substituent. $\mathrm{R}_{2}$ is the ribosyl-ADP moiety and $\mathrm{B}^{-}$is a general base.

anisms can be obtained from quantumchemical calculations of molecular properties along the reaction progress (usually the proton transfer coordinate). ${ }^{13}$ Nonadiabatic couplings ${ }^{15}$ and dipole moments ${ }^{24}$ are wellbehaved properties. But, the calculation of partial charges and spins, ${ }^{25}$ the simplest and most common diagnostic used, ${ }^{13,26-28}$ can be problematic because these are not physical observables and may be calculated with different recipes. ${ }^{29-31}$ Realizing these difficulties, intrinsic bond orbitals ${ }^{32}$ were recently proposed and successfully applied to identify proton and electron donors in PCET. ${ }^{33,34}$

Here we address the PCET mechanism in flavin mediated oxidation of NADH (reduced nicotinamide adenine dinucleotide) and of succinate catalyzed respectively by respiratory complex I (or type I NADH dehydrogenase) ${ }^{35-37}$ and by complex II (or succinate dehydrogenase, see Fig. 1). ${ }^{38,39}$ These fundamental steps for cellular respiration have been traditionally assigned to hydride transfers, ${ }^{36,39}$ but recent studies disputed this view and proposed HAT mechanisms. ${ }^{26,27}$

\section{Computational Methods}

The three reactions studied here are models of flavin-mediated oxidations catalyzed by respiratory complexes I and II (Fig. 1). The flavin isoalloxazine ring was represented by lumiflavin (LF, IUPAC name 7,8,10trimethylbenzo[g]pteridine-2,4-dione) instead of flavin mononucleotide (FMN) and flavin adenine dinucleotide (FAD) natural cofactors. Ribityl-ADP (adenosine diphosphate) and ribityl-phosphate moieties were replaced by methyl groups. The ribosyl-ADP moiety in NADH was replaced by a methyl group, generating 1-methylnicotinamide in the reduced form (MNAH or 1-methyl-4H-pyridine3-carboxamide, Fig. 1). Succinate had its carboxylates protonated into succinic acid for both E1 elimination from $\mathrm{H}_{2}$ Suc (butanedioic acid) and E1cb elimination from $\mathrm{H}_{2} \mathrm{Suc}^{-}[(\mathrm{E})-$ 4-hydroxy-4-oxidobut-3-enoic acid]. This is consistent with the enzyme active site in respiratory complex II, ${ }^{38}$ that shields the negative charges in succinate carboxylates by hydrogen bonding with positively charged residues, and with previous calculations on similar reactions. ${ }^{40}$ These models had 45,44 and 51 atoms, respectively.

Molecular geometries were optimized at the MP2 level ${ }^{41}$ with def2-SVP basis set. ${ }^{42}$ Resolution of identity with the def2 $/ \mathrm{J}^{43}$ and def2$\mathrm{SVP} / \mathrm{C}^{44}$ auxiliary basis was used. Optimizations with methods neglecting dispersion interactions resulted in disruption of the experimental ${ }^{35,38}$ stacked orientation between reactants. Transition structures were confirmed by analysis of the hessian eigenvector with negative eigenvalue and of the intrinsic reaction coordinates (Table S1 in Supporting In- 
formation). ${ }^{45}$

A reaction coordinate $\mathrm{RC}=r_{1}-r_{2}$ was defined by the broken bond distance $\left(r_{1}\right.$, between the transferred $\mathrm{H}$ and $\mathrm{C} 2$ atom in $\mathrm{H}_{2} \mathrm{Suc} / \mathrm{H}_{2} \mathrm{Suc}^{-}$or $\mathrm{C} 4$ atom in $\mathrm{MNAH}$ ) minus the formed bond distance $\left(r_{2}\right.$, between $\mathrm{H}$ and $\mathrm{N} 5$ atom in LF, Fig. 1). The minimum energy path connecting reactants and products was obtained with constrained geometry optimizations fixing $r_{1}$ while relaxing all others degrees of freedom using the ORCA 4.1.1 program. ${ }^{46}$ Intrinsic Bond Orbitals (IBOs) ${ }^{32}$ were obtained from localization $^{33}$ of the broken-symmetry solution ${ }^{34}$ of unrestricted ${\mathrm{M} 06^{47}}^{4 i n g l e-p o i n t ~ c a l c u l a t i o n s ~}$ with ORCA.

Complete active space self-consistent field $(\mathrm{CASSCF})^{28,41}$ were performed with the PySCF package version $1.5^{48}$ for singlet spin states in $\mathrm{C}_{1}$ point-group symmetry. All single-point calculations used the def2-TZVP basis set. Configuration and population analysis on CASSCF wave-functions were done with localized orbitals by the Pipek-Mezey scheme, while Foster-Boys localization led to equivalent results. ${ }^{49}$ This analysis was also performed on CASSCF/def2-SVP wavefunctions and similar results were observed. Active spaces contained 6 electrons in $5 \mathrm{MOs}$ (6e,5o) for $\mathrm{H}_{2} \mathrm{Suc},(8 \mathrm{e}, 6 \mathrm{o})$ for $\mathrm{H}_{2} \mathrm{Suc}^{-}$and $(12 \mathrm{e}, 10 \mathrm{o})$ for MNAH reactions as determined from analysis of occupation numbers and selection of reactive MOs. As MOs may change shape and ordering along the reaction path, CASSCF calculations started from the geometry with highest multiconfigurational character (product for $\mathrm{H}_{2} \mathrm{Suc}$, and reactant for $\mathrm{H}_{2} \mathrm{Suc}^{-}$and MNAH reactions) and guess MOs were taken from the CASSCF calculation of an adjacent geometry along the path.

The short-range influence of the enzymatic environment on the mechanism of flavinmediated oxidation was emulated with truncated active site models (Fig. S1 in Supporting Information). For succinate oxidation catalyzed by respiratory complex II, the model contained 192 atoms including FAD (for which the ribytil-ADP moiety was replaced by 2-hydroxyethyl), succinate (deprotonated, Suc), side chains of H365, T377,
E378, R402, H504 and R544, backbones of G170 and G546, and 8 water molecules, which compose the first coordination shell around the flavin and substrate groups (Fig. S1). The initial model structure was based on the $\mathrm{PDB}$ ID $1 \mathrm{Y} 0 \mathrm{P}^{38}$ from $F$. frigidimarina fumarate reductase flavoprotein, homologous to the respiratory complex II. This complete enzyme bound to succinate was solvated, neutralized and equilibrated for 22 ns of classical molecular dynamics simulation, using the CHARMM36 force field. ${ }^{50}$ The listed groups were extracted from this equilibrated structure and dangling covalent bonds were saturated by hydrogen atoms. Reactive geometries were obtained by relaxed potential energy surface scans along the same reaction coordinate defined in the main text $(\mathrm{RC})$ with position restrains to $\mathrm{C}_{\alpha}$ or $\mathrm{C}_{\beta}$ of capped aminoacids to preserve the active site architecture. This corresponds to the $\mathrm{H}_{2} \mathrm{Suc}$ reaction in the main text (Fig. 2B).

For NADH oxidation catalyzed by respiratory complex I, the active site model contained 167 atoms including FMN, NADH (for which phosphate group and ADP moiety were replaced by methyl groups), side-chains of residues N92, E97, Y180, N220, S295 and T325 and backbone of residues G183 and E184 from the Nqo1 subunit of $T$. thermophilus complex I as found in the PDB ID 3 IAM $^{35}$ structure. This model corresponds to the MNAH reaction in the main text (Fig. 2A). Geometries of stationary points were optimized in the B3LYP-D3/def2-SVP level ${ }^{51}$ and kindly provided by Prof. Ville Kaila from their original study. ${ }^{27}$ Geometries for the complex II model were optimized at the M06-L/def2-SVP level. ${ }^{52}$ Geometries of the enzyme models showed little dependency on the optimization level if dispersion interactions were accounted for (as in B3LYPD3 or M06-L functionals). Wave-functions for stationary points were obtained at the CASSCF/def2-SVP level with the same active spaces used for the isolated reactions. 


\section{Results \& Discussion}

Three model reactions corresponding to steps (a)-(c) in Fig. 1 were studied with lumiflavin (LF, $R_{1}=$ methyl) acceptor, NADH modeled as 1-methylnicotinamide (MNAH) and succinate protonated to $\mathrm{H}_{2} \mathrm{Suc}$ (succinic acid) for E1 elimination and $\mathrm{H}_{2} \mathrm{Suc}^{-}$(carbanion $\leftrightarrow$ enolate electromer) for E1cb elimination. See panels $\mathrm{A}, \mathrm{B}$ and $\mathrm{C}$ in Fig. 2 for the reactant structures, consistent with enzymatic active sites.

In order to quantify the extent of electron transfer along the reaction and avoid the pitfalls of using only partial charges to analyze PCET mechanisms, ${ }^{29,33}$ we propose a simple quantitative diagnostic of electron transfer employing weights of wave-function configurations constructed with orbitals localized in the donor. This electronic charge transferred, $\Delta o x i$, is defined along the reaction coordinate $(\mathrm{RC})$ as:

$$
\Delta o x i(R C)=\sum_{n} n w_{n}(R C)-\max \{n\}
$$

where $n$ is the number of electrons occupying active MOs localized in the donor, $\max \{n\}$ is their maximum occupation at the reactant state and $w_{n}$ is the combined weight of wave-function configurations with $n$-electrons. Only configurations with $w_{n}>$ $1 \%$ in at least one geometry along the reaction were considered. This analysis may be used with any kind of multiconfigurational wavefunction obtained with localized MOs. ${ }^{41}$

We start with the $\mathrm{H}_{2} \mathrm{Suc}$ reaction (model for succinate E1 elimination, Fig. 2B) in which the broken $\mathrm{C}-\mathrm{H}$ bond $\sigma$ MO becomes a non-bonding $\mathrm{MO}$ in the product. This is the only active MO localized in the $\mathrm{H}_{2}$ Suc donor, thus $\{n\}=(0,1,2)$ electrons and $\max \{n\}=$ 2. Fig. $2 \mathrm{~B}$ middle column shows that the contribution (combined weight) to the wavefunction of this localized MO changes from almost $100 \%$ for 2 -electron occupation in the reactant to $80 \%$ for 1-electron occupation in the product. The average electronic charge transferred is $\Delta o x i=-1$, showing the $\mathrm{H}_{2} \mathrm{Suc}$ reaction is a hydrogen atom transfer (HAT).

In the bi-radical product, an unpaired elec- tron occupies the LF $\pi^{*} \mathrm{MO}$ and spin-couples to a singlet state with the unpaired electron in the carbocation (Fig. 2B top of middle column), resulting in a short distance $\left(r_{1}=2.3 \AA\right)$ between the two molecules.

Partial charges condensed on the LF and $\mathrm{H}_{2} \mathrm{Suc}$ groups are similar (within $0.2 e$, Fig. $2 \mathrm{~B}$ right column) and nearly neutral when comparing reactant and product (end) states, suggesting there is no net charge separation or redistribution, in accordance to a HAT. ${ }^{13,15}$ The partial charge on the transferred $\mathrm{H}$ atom is close to $0.2 e$ along the complete pathway for all reactions studied here (Fig. 2 right column). A naive interpretation of this partial charge would suggest a HAT is observed in all reactions, but this is not the case as clearly shown for the other two reactions below.

For $\mathrm{H}_{2} \mathrm{Suc}^{-}$(E1cb elimination, Fig. 2C), the non-bonding $\mathrm{MO}$ with the extra electron lone-pair and the $\sigma \mathrm{MO}$ of the broken $\mathrm{C}-\mathrm{H}$ bond localize in the donor. Only configurations with $\{n\}=(2,3,4)$ electrons in these orbitals contribute significantly (more than $1 \%$ ) to the wave-function, thus $\max \{n\}=$ 4. Remarkably, the anionic reactant already shows a substantial charge transfer $(\Delta o x i=$ -0.5 at $\mathrm{RC}=-1.0 \AA)$. When fumaric acid is formed, only configurations with 2 electrons in the forming double bond become relevant, resulting in a much longer distance $\left(r_{1}=3.8 \AA\right)$ between $\mathrm{LF}^{-}$and fumaric acid. The average $\Delta o x i=-2$ in the product and this reaction is clearly a hydride transfer. Partial charges change considerably between end states and LF has a $-1 e$ charge in the product, as expected from receiving a hydride.

For the MNAH reaction (Fig. 2A), the $\mathrm{MO}$ of the broken $\mathrm{C}-\mathrm{H}$ bond becomes a $\pi$ $\mathrm{MO}$ and conjugates with the other nicotinamide MOs. A total of 6 active MOs are localized in the donor. Only configurations with $\{n\}=(6,7,8)$ electrons in these orbitals contribute significantly to the wave-function, thus $\max \{n\}=8$. Weights change from almost $100 \%$ for configurations with 8 electrons in the reactant state to $85 \%$ for 6 electrons in the product, with a final $\Delta o x i \simeq-2$. We conclude this reaction is also a hydride trans- 

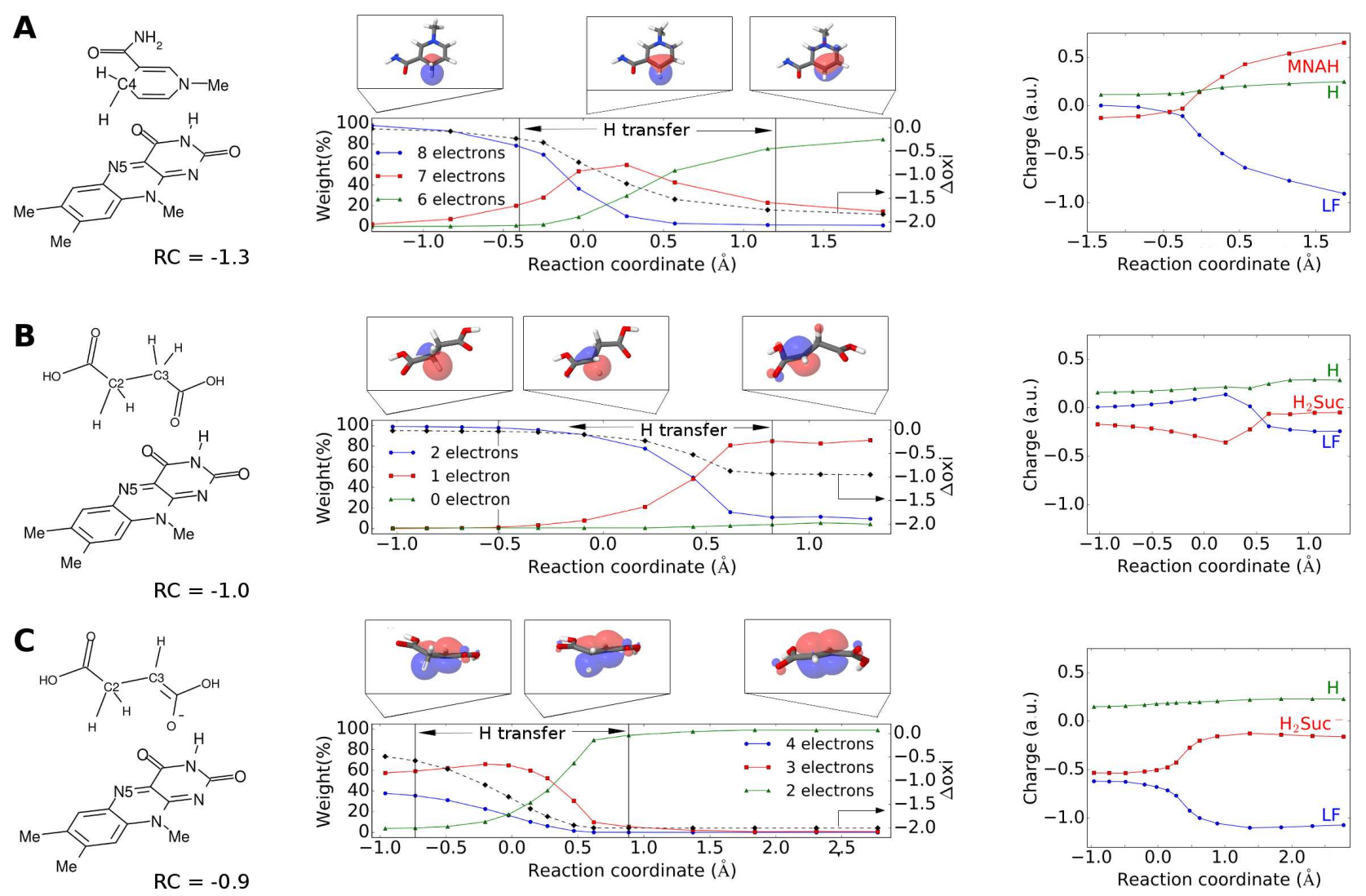

Figure 2: Mechanisms of flavin mediated oxidation from analysis of the wave-function along the reaction. Panels $\mathrm{A}, \mathrm{B}$ and $\mathrm{C}$ show the reactions studied here, corresponding respectively to steps (a), (b) and (c) in Fig. 1. The left column presents the reactant states. The middle column shows the contribution or weight of wave-function configurations with $n$-electrons in molecular orbitals (MOs) localized in the donor along the proton-transfer reaction coordinate. $\Delta o x i$ is calculated from equation 1 and shown in dashed line. In the $\mathrm{H}$ transfer range, the transferred hydrogen is not fully bound to either donor or acceptor. The broken $\mathrm{C}-\mathrm{H}$ bond $\sigma$ MO is shown on top, and the the lone-pair MO is also shown for $\mathrm{H}_{2} \mathrm{Suc}^{-}$. The right column shows Mulliken partial charges for the transferred $\mathrm{H}$, flavin and donor groups.

fer.

It is noteworthy that weights for configurations with an odd-number of electrons localized in the donor ( 1 electron for $\mathrm{H}_{2} \mathrm{Suc}$, 3 for $\mathrm{H}_{2} \mathrm{Suc}^{-}$and 7 for MNAH reactions) peak near the transition state (TS, RC=0.03 Åfor MNAH, 0.61 Åfor $\mathrm{H}_{2}$ Suc and 0.14 Afor $\mathrm{H}_{2} \mathrm{Suc}^{-}$, Table S1). This is in line with $\Delta o x i \simeq-1$ at the TS for the three reactions (Fig. 2 middle column) and suggests that the activation energies correspond to the first (and only in $\mathrm{H}_{2} \mathrm{Suc}$ reaction) electron transfer. Partial charge or other population analysis $^{30-32}$ can not usually provide this level of detail.

Electron and proton transfer occur concerted along the same range of reaction coordinate for the three studied reactions $(\mathrm{H}$ transfer indicated in Fig. 2). However, analysis of intrinsic bond orbitals (IBO, ${ }^{32,33}$ Fig. 3) suggests the two transfers take place from (or to) the same donor (acceptor) site only for the MNAH reaction as orbitals (and the corresponding electron density) from the $\mathrm{C}-$ $\mathrm{H}$ bond in the reactant turn into the $\mathrm{N} 5-\mathrm{H}$ bond in the product after $\mathrm{H}^{-}$transfer. On the other hand, electron and proton transfer in the two succinic acid reactions proceed to different sites of the acceptor flavin. These two reactions should be classified as concerted $\mathrm{PCET}^{11,13}$ or multiple-site $\mathrm{PCET}^{14}$ as orbitals of the broken $\mathrm{C}-\mathrm{H}$ bond in the reactant do not turn into the $\mathrm{N} 5-\mathrm{H}$ bond in the product, and either delocalize over the flavin pyrimidinedione ring $\left(\mathrm{H}_{2} \mathrm{Suc}\right.$ reaction) or form the double bond in fumaric acid 


\section{$\left(\mathrm{H}_{2} \mathrm{Suc}^{-}\right)$.}

Finally, we tested whether the mechanisms observed above are conserved in the enzymatic environment. Truncated active site models with 167 and 192 atoms respectively coordinating NADH in respiratory complex I and succinate in respiratory complex II were built from these protein crystallographic structures (Fig. S1). ${ }^{35,37,38}$ Analysis of $\Delta o x i$ (equation 1) calculated for these active site models using the same methods depicted above shows NADH oxidation proceeds via hydride transfer and succinate oxidation in E1 elimination occurs through HAT (Table S2), showing the enzymatic mechanisms are equivalent to those of the isolated models.

\section{Conclusions}

We confirm the traditional view ${ }^{36}$ that $\mathrm{NADH}$ oxidation by flavin proceeds via a prototypical hydride transfer, with the two electrons moving concerted with the proton from donor to the same acceptor group. A previous proposal of HAT for this reaction ${ }^{27}$ is incorrect and illustrates the pitfalls of assigning PCET mechanisms with diagnostics based only on partial charges. For succinate oxidation by flavin, two reaction sequences are possible. E1 elimination may occur by a HAT, opposed to usual proposals, ${ }^{39}$ but E1cb elimination will also proceed via hydride with advanced charge transfer in the reactant state.

PCET mechanisms of flavin mediated oxidation depend on the donor molecule. This should have implications for the mechanisms of several flavoproteins besides those of the respiratory chain studied here. It is also expected that the simple diagnostic of electron transfer along the reaction pathway proposed here will be useful to characterize a broad class of PCET processes.

Acknowledgement Fruitful discussions with Prof. Peter R. Taylor and funding from FAPESP (scholarships 2016/235250 and 2017/26109-0 to F.C., and grants 2016/24096-5 and 2019/21856-7 to G.M.A.) are gratefully acknowledged.

\section{Supporting Information Available}

One figure with the structure of truncated enzyme active site models, and two tables with quantification of electron transfer $(\Delta o x i)$ for enzyme models and TS analysis

\section{References}

(1) Joosten, V.; van Berkel, W. J. Flavoenzymes. Curr. Op. Chem. Biol. 2007, 11, 195-202.

(2) Sobrado, P.; Gadda, G. Introduction to Flavoproteins: Beyond the Classical Paradigms. Arch. Biochem. Biophys. 2017, 632, $1-3$.

(3) Gray, M.; Goodman, A. J.; Carroll, J. B.; Bardon, K.; Markey, M.; Cooke, G.; Rotello, V. M. Model Systems for Flavoenzyme Activity: Interplay of Hydrogen Bonding and Aromatic Stacking in Cofactor Redox Modulation. Org. Lett. 2004, 6, 385-388.

(4) Nandwana, V.; Samuel, I.; Cooke, G.; Rotello, V. M. Aromatic Stacking Interactions in Flavin Model Systems. Acc. Chem. Res. 2013, 46, 1000-1009.

(5) Reis, A. A. O.; Sayegh, R. S. R.; Marana, S. R.; Arantes, G. M. Combining Free Energy Simulations and NMR Chemical-Shift Perturbation To Identify Transient Cation- $\pi$ Contacts in Proteins. J. Chem. Inf. Model. 2020, 60, 890-897.

(6) Chaiyen, P.; Scrutton, N. S. Flavins and Flavoproteins. FEBS J. 2015, 282, 3001-3002.

(7) Bresnahan, C. G.; Reinhardt, C. R.; Bartholow, T. G.; Rumpel, J. P.; North, M.; Bhattacharyya, S. Effect of Stacking Interactions on the Thermodynamics and Kinetics of Lumiflavin: 
A
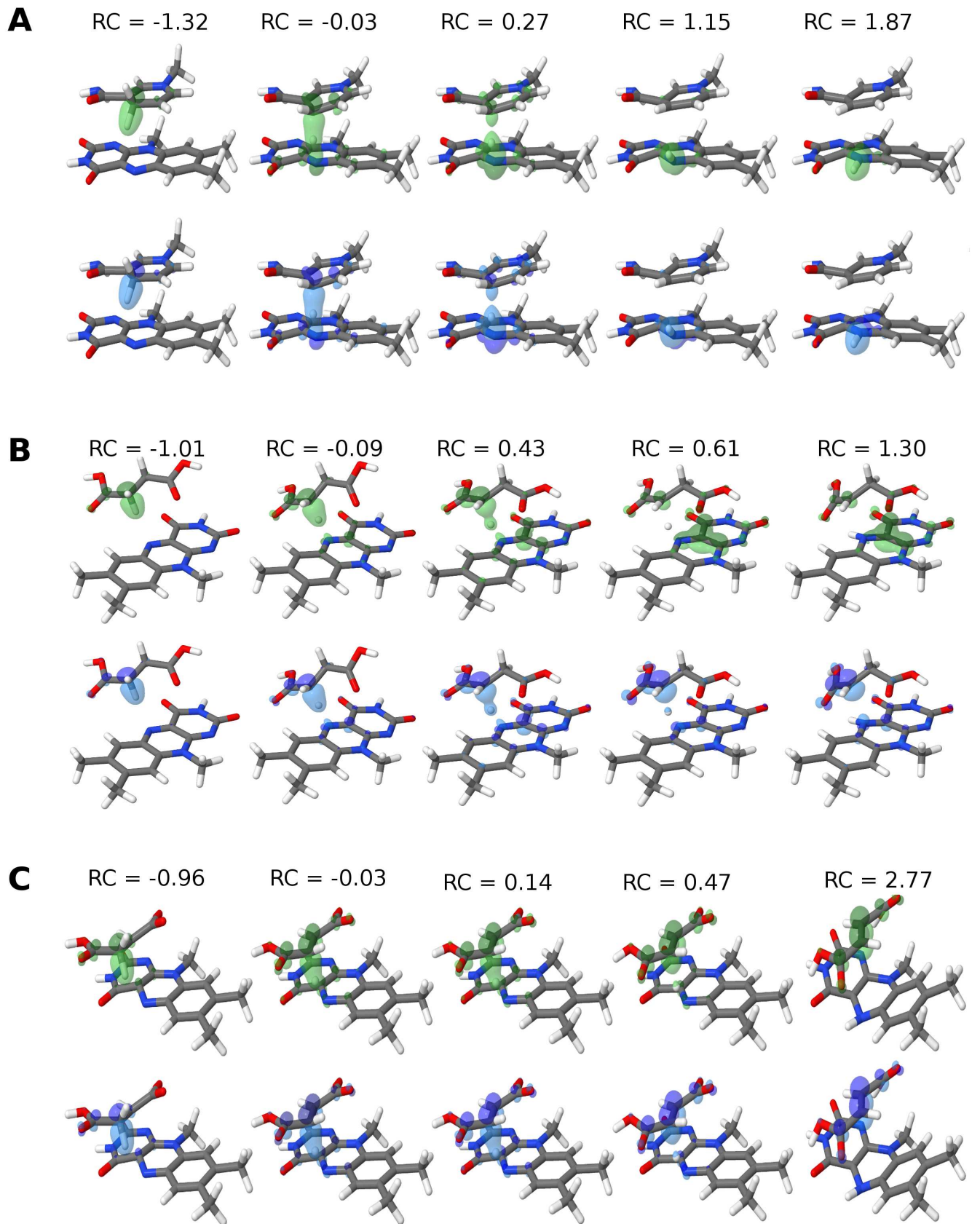

Figure 3: Intrinsic bond orbitals of the broken $\mathrm{C}-\mathrm{H}$ bond for geometries with a given reaction coordinate (RC) along the pathway for MNAH (A), $\mathrm{H}_{2} \mathrm{Suc}(\mathrm{B})$ and $\mathrm{H}_{2} \mathrm{Suc}^{-}(\mathrm{C})$ oxidation. Green $\alpha$ and blue $\beta$ spin-orbitals are shown.

A Study with Improved Density Functionals and Density Functional TightBinding Protocol. J. Phys. Chem. A 2015, 119, 172-182.

(8) Buckel, W.; Thauer, R. K. Flavin-Based Electron Bifurcation, A New Mechanism of Biological Energy Coupling. Chem. Rev. 2018, 118, 3862-3886.

(9) Cukier, R. I.; Nocera, D. G. ProtonCoupled Electron Transfer. Annu. Rev. Phys. Chem. 1998, 49, 337-369.
(10) Huynh, M. H. V.; Meyer, T. J. ProtonCoupled Electron Transfer. Chem. Rev. 2007, 107, 5004-5064.

(11) Warren, J. J.; Tronic, T. A.; Mayer, J. M. Thermochemistry of Proton-Coupled Electron Transfer Reagents and its Implications. Chem. Rev. 2010, 110, 6961-7001.

(12) Hammes-Schiffer, S. Comparison of Hydride, Hydrogen Atom, and Proton- 
Coupled Electron Transfer Reactions. ChemPhysChem 2002, 3, 33-42.

(13) Hammes-Schiffer, S. Proton-Coupled Electron Transfer: Classification Scheme and Guide to Theoretical Methods. Energy Environ. Sci. 2012, 5, 7696-7703.

(14) Darcy, J. W.; Koronkiewicz, B.; Parada, G. A.; Mayer, J. M. A Continuum of Proton-Coupled Electron Transfer Reactivity. Acc. Chem. Res. 2018, 51, 2391-2399.

(15) Hammes-Schiffer, S.; Stuchebrukhov, A. A. Theory of Coupled Electron and Proton Transfer Reactions. Chem. Rev. 2010, 110, 6939-6960.

(16) Brenner, S.; Hay, S.; Heyes, D. J.; Scrutton, N. S. Proton-Coupled Electron Transfer: A Carrefour of Chemical Reactivity Traditions; The Royal Society of Chemistry, 2012; pp 57-88.

(17) Poulsen, T. D.; Garcia-Viloca, M.; Gao, J.; Truhlar, D. G. Free Energy Surface, Reaction Paths, and Kinetic Isotope Effect of Short-Chain AcylCoA Dehydrogenase. J. Phys. Chem. B 2003, 107, 9567-9578.

(18) Bhattacharyya, S.; Stankovich, M. T.; Truhlar, D. G.; Gao, J. Combined Quantum Mechanical and Molecular Mechanical Simulations of One- and Two-Electron Reduction Potentials of Flavin Cofactor in Water, MediumChain Acyl-CoA Dehydrogenase, and Cholesterol Oxidase. J. Phys. Chem. A 2007, 111, 5729-5742.

(19) Pang, J.; Hay, S.; Scrutton, N. S.; Sutcliffe, M. J. Deep Tunneling Dominates the Biologically Important $\mathrm{Hy}-$ dride Transfer Reaction from NADH to FMN in Morphinone Reductase. J. Am. Chem. Soc. 2008, 130, 7092-7097.

(20) Delgado, M.; Grlich, S.; Longbotham, J. E.; Scrutton, N. S.;
Hay, S.; Moliner, V.; Tun, I. Convergence of Theory and Experiment on the Role of Preorganization, Quantum Tunneling, and Enzyme Motions into Flavoenzyme-Catalyzed Hydride Transfer. ACS Catal. 2017, 7, 3190-3198.

(21) Tan, S. L. J.; Novianti, M. L.; Webster, R. D. Effects of Low to Intermediate Water Concentrations on ProtonCoupled Electron Transfer (PCET) Reactions of Flavins in Aprotic Solvents and a Comparison with the PCET Reactions of Quinones. J. Phys. Chem. B 2015, 119, 14053-14064.

(22) Mathes, T.; Zhu, J.; van Stokkum, I. H. M.; Groot, M. L.; Hegemann, P.; Kennis, J. T. M. Hydrogen Bond Switching among Flavin and Amino Acids Determines the Nature of ProtonCoupled Electron Transfer in BLUF Photoreceptors. J. Phys. Chem. Lett. 2012, 3, 203-208.

(23) Goings, J. J.; Hammes-Schiffer, S. Early Photocycle of Slr1694 Blue-Light Using Flavin Photoreceptor Unraveled through Adiabatic Excited-State Quantum Mechanical/Molecular Mechanical Dynamics. J. Am. Chem. Soc. 2019, 141, 20470-20479.

(24) Soudackov, A. V.; Hammes-Schiffer, S. Probing Nonadiabaticity in the ProtonCoupled Electron Transfer Reaction Catalyzed by Soybean Lipoxygenase. J. Phys. Chem. Lett. 2014, 5, 3274-3278.

(25) Sirjoosingh, A.; Hammes-Schiffer, S. Proton-Coupled Electron Transfer versus Hydrogen Atom Transfer: Generation of Charge-Localized Diabatic States. J. Phys. Chem. A 2011, 115, 2367-2377.

(26) Abe, Y.; Shoji, M.; Nishiya, Y.; Aiba, H.; Kishimoto, T.; Kitaura, K. The Reaction Mechanism of Sarcosine Oxidase Elucidated Using FMO and QM/MM Methods. Phys. Chem. Chem. Phys. 2017, 19, 9811-9822. 
(27) Saura, P.; Kaila, V. R. I. Energetics and Dynamics of Proton-Coupled Electron Transfer in the NADH/FMN Site of Respiratory Complex I. J. Am. Chem. Soc. 2019, 141, 5710-5719.

(28) Arantes, G. M.; Bhattacharjee, A.; Field, M. J. Homolytic Cleavage of Fe-S Bonds in Rubredoxin Under Mechanical Stress. Angew. Chem. Int. Ed. 2013, 52, 8144-8146.

(29) Politzer, P.; Harris, R. R. Properties of Atoms in Molecules. I. Proposed Definition of the Charge on an Atom in a Molecule. J. Am. Chem. Soc. 1970, 94, 6451-6454.

(30) Reed, A. E.; Weinstock, R. B.; Weinhold, F. Natural Population Analysis. J. Chem. Phys. 1985, 83, 735-746.

(31) Martin, F.; Zipse, H. Charge Distribution in the Water Molecule - A Comparison of Methods. J. Comput. Chem. 2004, 26, 97-105.

(32) Knizia, G. Intrinsic Atomic Orbitals: An Unbiased Bridge between Quantum Theory and Chemical Concepts. $J$. Chem. Theory Comput. 2013, 9, 48344843.

(33) Klein, J. E. M. N.; Knizia, G. cPCET versus HAT: A Direct Theoretical Method for Distinguishing X-H BondActivation Mechanisms. Angew. Chem. Int. Ed. 2018, 57, 11913-11917.

(34) Mandal, M.; Elwell, C. E.; Bouchey, C. J.; Zerk, T. J.; Tolman, W. B.; Cramer, C. J. Mechanisms for Hydrogen-Atom Abstraction by Mononuclear Copper(III) Cores: Hydrogen-Atom Transfer or Concerted Proton-Coupled Electron Transfer? J. Am. Chem. Soc. 2019, 141, 1723617244.

(35) Berrisford, J. M.; Sazanov, L. A. Structural Basis for the Mechanism of Respiratory Complex I. J. Biol. Chem. 2009, 284, 29773-29783.
(36) Hirst, J. Mitochondrial Complex I. Annu. Rev. Biochem. 2013, 82, 551575.

(37) Teixeira, M. H.; Arantes, G. M. Balanced Internal Hydration Discriminates Substrate Binding to Respiratory Complex I. Biochim. Biophys. Acta 2019, 1860, 541-548.

(38) Wardrope, C.; Mowat, C. G.; Walkinshaw, M. D.; Reid, G. A.; Chapman, S. K. Fumarate Reductase: Structural and Mechanistic Insights from the Catalytic Reduction of 2methylfumarate. FEBS Lett. 2006, 580, 1677-1680.

(39) Maklashina, E.; Cecchini, G.; Dikanov, S. A. Defining a Direction: Electron Transfer and Catalysis in Escherichia coli Complex II Enzymes. Biochim. Biophys. Acta 2013, 182\%, 668-678.

(40) Lucas, M. F.; Ramos, M. J. Mechanism of a Soluble Fumarate Reductase from Shewanella frigidimarina: A Theoretical Study. J. Phys. Chem. B 2006, 110, 10550-10556.

(41) Helgaker, T.; Jørgensen, P.; Olsen, J. Molecular Electronic-Structure Theory, 1st ed.; Wiley: New York, 2000.

(42) Weigend, F.; Ahlrichs, R. Balanced Basis Sets of Split Valence, Triple Zeta Valence and Quadruple Zeta Valence Quality for $\mathrm{H}$ to Rn: Design and Assessment of Accuracy. Phys. Chem. Chem. Phys. 2005, 7, 3297-3305.

(43) Weigend, F. Accurate Coulomb-Fitting Basis Sets for $\mathrm{H}$ to Rn. Phys. Chem. Chem. Phys. 2006, 8, 1057-1065.

(44) Hellweg, A.; Hättig, C.; Höfener, S.; Klopper, W. Optimized Accurate Auxiliary Basis Sets for RI-MP2 and RI-CC2 Calculations for the Atoms $\mathrm{Rb}$ to $\mathrm{Rn}$. Theor. Chem. Acc. 2007, 117, 587-597. 
(45) Fukui, K. The Path of Chemical Reactions - The IRC Approach. Acc. Chem. Res. 1981, 14, 363-368.

(46) Neese, F. Software Update: The ORCA Program System, Version 4.0. WIREs Rev. Comput. Mol. Sci. 2018, 8, e1327.

(47) Zhao, Y.; Truhlar, D. G. The M06 Suite of Density Functionals for Main Group Thermochemistry, Thermochemical Kinetics, Noncovalent Interactions, Excited States, and Transition Elements: Two New Functionals and Systematic Testing of Four M06-Class Functionals and 12 Other Functionals. Theor. Chem. Acc. 2008, 120, 215-241.

(48) Sun, Q.; Berkelbach, T. C.; Blunt, N. S.; Booth, G. H.; Guo, S.; Li, Z.; Liu, J.; McClain, J. D.; Sayfutyarova, E. R.; Sharma, S.; Wouters, S.; Chan, G. K.-L. PySCF: The Python-based Simulations of Chemistry Framework. WIREs Rev. Comput. Mol. Sci. 2017, 8, e1340.

(49) Pipek, J.; Mezey, P. A Fast Intrinsic Localization Procedure Applicable for ab Initio and Semiempirical Linear Combination of Atomic Orbital Wave Functions. J. Chem. Phys. 1989, 90, 49164926.

(50) Huang, J.; MacKerell Jr, A. D. CHARMM36 All-Atom Additive Protein Force Field: Validation Based on Comparison to NMR Data. J. Comput. Chem. 2013, 34, 2135-2145.

(51) Becke, A. D. Density Functional Thermochemistry. III. The Role of Exact Exchange. J. Chem. Phys. 1993, 98, 5648.

(52) Zhao, Y.; Truhlar, D. G. A New Local Density Functional for MainGroup Thermochemistry, Transition Metal Bonding, Thermochemical Kinetics, and Noncovalent Interactions. $J$. Chem. Phys. 2006, 125, 194101. 


\section{Supporting Information}

\section{Mechanisms for Flavin Mediated Oxidation: Hydride or Hydrogen-Atom Transfer?}

Felipe Curtolo and Guilherme M. Arantes*

Department of Biochemistry, Instituto de Química, Universidade de São Paulo, Av. Prof. Lineu Prestes 748, 05508-900, São Paulo, SP, Brazil

E-mail: garantes@iq.usp.br
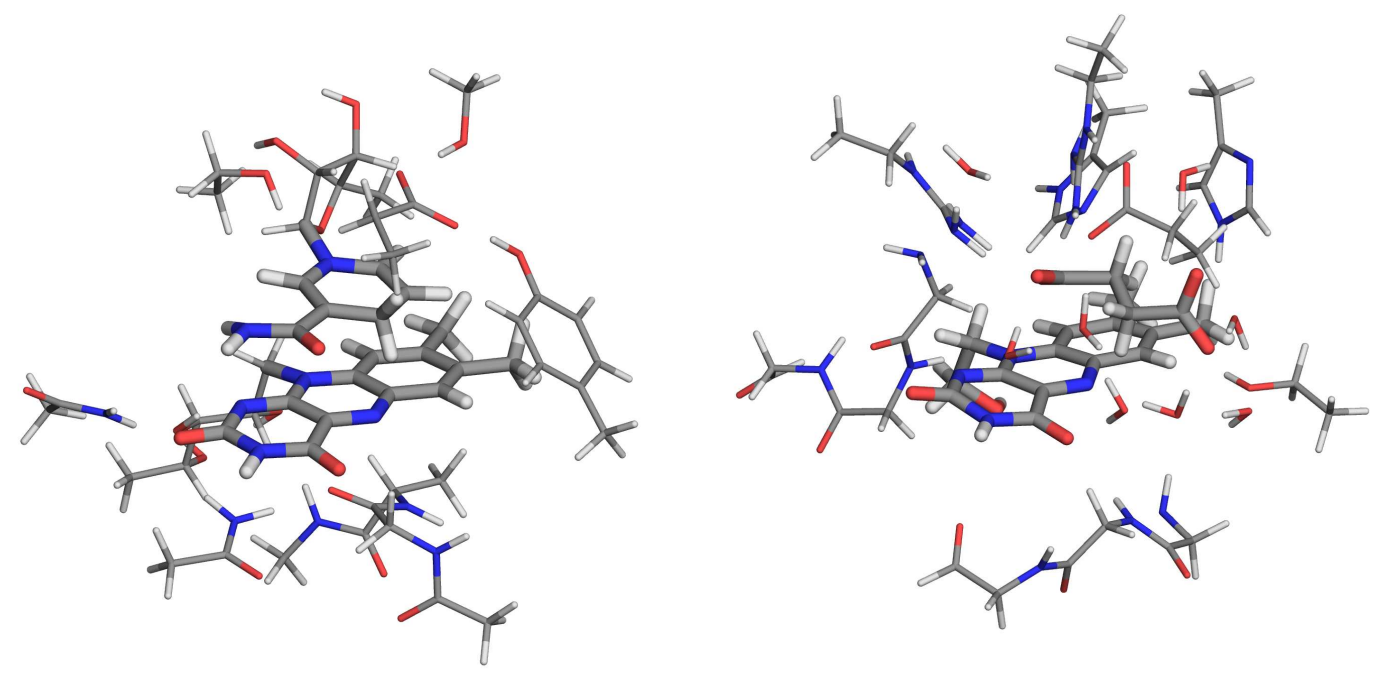

Figure S1: Structure of truncated active site models for respiratory complexes I (left) and II (right) used for calculations in Table S2. Flavin, the nicotinamide ring of NADH (left) and succinate (right) are shown in thick sticks. 
Table S1: Reaction coordinates and mode component analysis for transition states (TS) of flavin mediated oxidation.

\begin{tabular}{l|cccc}
\hline \hline Donor & $r_{1}(\AA)$ & $r_{2}(\AA)$ & RC $(\AA)$ & Frequency $\left(\mathrm{cm}^{-1}\right)$ \\
\hline MNAH & 1.30 & 1.33 & -0.03 & -859.2 \\
$\mathrm{H}_{2} \mathrm{Suc}$ & 1.68 & 1.07 & 0.61 & -827.1 \\
$\mathrm{H}_{2} \mathrm{Suc}^{-}$ & 1.40 & 1.26 & 0.14 & -947.0 \\
\hline \hline
\end{tabular}

Table S2: Average number of electrons transferred ( $\Delta$ oxi, equation 1 in the main text) and reaction coordinate ( $\mathrm{RC}$ in $\AA$ ) for stationary points of flavin mediated oxidation in active site models of the respiratory complex I reaction with NADH and complex II reaction with succinate via E1 elimination (Fig. S1).

\begin{tabular}{l|lc||lc}
\hline \hline & \multicolumn{2}{|l||}{ Complex I + NADH } & \multicolumn{2}{c}{ Complex II + succinate } \\
& $\Delta$ oxi & RC & $\Delta o x i$ & RC \\
\hline Reactant & -0.06 & -1.00 & -0.02 & -1.67 \\
TS & -0.93 & 0.00 & -1.00 & 1.36 \\
Product & -1.92 & 1.86 & -1.00 & 1.70 \\
\hline \hline
\end{tabular}

